\title{
Does Nonalcoholic Pancreatic Steatosis Always Correlate with Nonalcoholic Fatty Liver Disease?
}

\author{
Celal Ulasoglu (D) \\ Zeynep Nilufer Tekin ${ }^{2}$ \\ Kubra Akan' \\ Arda Yavuz $\mathbb{D D}^{\prime}$ \\ 'Department of Gastroenterology, \\ Medeniyet University, Goztepe Education \\ and Research Hospital, Istanbul, Turkey; \\ ${ }^{2}$ Department of Radiology, Medeniyet \\ University, Goztepe Education and \\ Research Hospital, Istanbul, Turkey
}

Purpose: To identify the correlation of nonalcoholic pancreatic steatosis (NAPS) with nonalcoholic fatty liver disease (NAFLD) in an outpatient group. Based on its metabolic and imaging properties, NAPS has been increasingly recognized in recent years; however, its interaction with NAFLD is still not clear.

Patients and Methods: In this cross-sectional observational study, 345 consecutive patients without any chronic illness who were referred to the senior radiologist for abdominal ultrasound (US) were included. The US report showed hepatic and pancreatic echogenicity. The patients' demographic, anthropometric, and laboratory data were collected from medical records.

Results: Overall, NAPS and NAFLD were seen in 227 (65.8\%) and 219 (63.5\%) patients, respectively. Normal echogenicity was noted in $74(21.4 \%)$ patients. Forty-four patients $(12.8 \%)$ had steatotic liver without NAPS, 52 (15.1\%) had steatotic pancreas without NAFLD, and $175(50.7 \%)$ had steatosis in both organs. The discordance in steatosis grading between NAPS and NAFLD was 55.1\%. Insulin resistance was present in 8.7, 26.7, 19, and $61.3 \%$ of patients with no steatosis, only NAFLD, only NAPS, and steatosis in both organs, respectively. Evident NAFLD and NAPS having grade 2 and 3 steatosis were present in $15.3 \%$ and $29.0 \%$ of the study group, respectively. Cholecystolithiasis was present in 6.8 , 13.6 , and $28.8 \%$ of patients with normal echogenic pancreas, only NAFLD, and only NAPS, respectively $(\mathrm{p}=0.01)$.

Conclusion: Based on the ultrasonographic, clinical, demographic, and anthropometric features of the included patients, we found that NAPS did not fully accompany nonalcoholic fatty liver. Despite severe pancreatic steatosis, more than a quarter of cases had normal liver echogenicity. Insulin resistance frequency was insignificantly higher in NAFLD than NAPS $(p=0.694)$. The significantly higher frequency of cholecystolithiasis in NAPS needs further large-scale studies. The inconsistency of steatosis degree in NAPS and NAFLD in $>50 \%$ cases may reflect differences in the pathophysiology of these two clinical entities.

Keywords: nonalcoholic pancreatic steatosis, nonalcoholic fatty liver disease, insulin resistance, ultrasound

\section{Introduction}

Nonalcoholic fatty liver (NAFLD) is defined as the intracellular deposition of triglycerides in hepatocytes, and nonalcoholic pancreatic steatosis (NAPS) is identified as the fatty infiltration of the pancreas; both conditions were found to be highly related with metabolic, cardiovascular, and oncological risks, as well as obstructive sleep apnea. ${ }^{1-4}$ Fat replacement of the pancreas is substitution of fat in areas of acinar cell loss, while fat accumulation is parallel to an increase in total body adipocyte load leading to fatty infiltration of the pancreas. ${ }^{1,2}$ Despite being a fairly new disease entity,
Correspondence: Celal Ulasoglu Medeniyet University, Goztepe Education and Research Hospital, Istanbul, Turkey

Tel +902165666600

Fax +902166065210

Email ulasoglu@gmail.com 
there are approximately 5346 publications on NAPS in PubMed, as revealed by the keyword search on "pancreatic steatosis", accessed on April 16, 2021; in contrast, NAFLD featured in a total of 91,386 publications. NAPS is generally regarded as a consequence of NAFLD. ${ }^{1,6,7}$ The relationship of NAPS with the severity of acute pancreatitis and development of pancreatic cancer is still debatable. ${ }^{1,2}$ In this study, we aimed to compare the features, correlations, and inconsistencies of NAPS and NAFLD. Fat infiltration of the pancreas was assessed in $35 \%$ of medical check-up cases. ${ }^{8}$

\section{Patients and Methods}

In this retrospective, observational, analytical case-control study, the cases comprised 345 consecutive dyspeptic patients without any chronic illnesses, who were referred for abdominal ultrasound (US) to the senior radiologist blinded to the clinical and laboratory details. The report included the height and contour of the liver, portal vein wall visualization, gall bladder examination, echogenicity status, and grading of hepatic and pancreatic steatosis. Pancreatic echogenicity was evaluated using the following grading system: Grade $0=$ pancreatic echogenicity was equal to renal cortical echogenicity, Grade $1=$ definitely lower than retroperitoneal fat, Grade $2=$ slightly lower than retroperitoneal fat, and Grade $3=$ equal to retroperitoneal fat. The grading for NAFLD was as follows: Grade $0=$ normal echotexture of the liver, Grade $1=$ slight and diffuse increase of liver echogenicity with normal visualization of the diaphragm and portal vein wall, Grade $2=$ moderate increase of liver echogenicity with impaired appearance of the portal vein wall but still appreciable diaphragm echogenicity, and Grade 3=marked increase of liver echogenicity with almost no visualization of the portal vein wall, diaphragm, and posterior part of the right liver lobe. ${ }^{9-11}$

Body mass index (BMI) was calculated as weight $(\mathrm{kg}) /$ square of height (m). A BMI between 17 and $25 \mathrm{~kg} / \mathrm{m}^{2}$ was accepted as the normal range. Body fat percentage (BFP) was estimated using the following formula: $\mathrm{BFP}=1.39 \times \mathrm{BMI}+(0.16 \times$ age $) \times(10.34 \times$ sex $)-9$, where $\operatorname{sex}$ for female was assigned a 0 and for male was assigned a 1 .

The demographic, anthropometric, and laboratory data from medical records of patients were also collected. Study approval was obtained from the ethical committee at Medeniyet University (2019-015). The tenets of the Declaration of Helsinki and guidelines of Good Clinical Practice were adhered to. The need for informed consent was waived because of the retrospective nature of the study. The authors preserved the privacy and confidentiality of study cases.

\section{Statistical Analysis}

All statistics were performed in SPSS 20 (IBM Corporation, Armonk, NY, USA). Numerical data are given as mean \pm standard deviation $(m e a n+S D)$. The power analysis was estimated with effect size 0.2 , alpha 0.05 , power 0.80 , and $\mathrm{dF}$ as 5 , which gave an output of 321 cases. The normality of distribution was assessed by Kolmogorov-Smirnov and Shapiro-Wilk tests depending on case number. Categorical data were analyzed by chisquare or Fisher's exact tests. Student's $t$-test or MannWhitney $U$ test were used for comparison of two groups, and ANOVA or Kruskal-Wallis were used for $>2$ groups depending on normality of distribution. The cut-off point was calculated based on a receiver operator characteristic (ROC) curve analysis. A Spearman correlation test with a binary logistic regression test was performed to analyze the correlation between parametric data. The results were given as mean $\pm \mathrm{SD} . \mathrm{P}<0.05$ was considered to indicate statistical significance.

\section{Results}

The study group consisted of 345 patients (220 female [mean age: $50 \pm 15$ years, range: 19-84] and 125 male [mean age: $52 \pm 15$ years, range: $20-84]$ ]). Normal liver and pancreatic echogenicity was present in only $74(21.4 \%)$, NAPS with normal liver was present in 52 (15.1\%), NAFLD with normal pancreas was detected in 44 (12.8\%), and steatosis in both liver and pancreas was seen in $175(50.7 \%)$ patients. The discordance in steatosis grading between NAPS and NAFLD was $55.1 \%$. Isolated steatosis of liver or pancreas was assessed in 96 (35.4) of 271 cases with NAPS and NAFLD, whereas 175 (64.6\%) of 271 patients had steatosis in both organs (Table 1). Insulin resistance (IR) was present in 8.7, 26.7, 19, and $61.3 \%$ of cases with no steatosis, only NAFLD, only NAPS, and both steatotic organs, respectively. Cholecystolithiasis, sludge, or history of cholecystectomy were noted in 6.8, 13.6, 28.8 , and $28.3 \%$ of cases with no steatosis, only NAFLD, only NAPS, and both steatotic organs steatotic, respectively $(\mathrm{p}=0.01)$. Evident NAFLD and NAPS with grade 2 or grade 3 steatosis was present in $15.3 \%$ and $29.0 \%$ of patients, respectively. Cases with only NAFLD and only NAPS had insignificant differences in older age, IR, total cholesterol, LDL-cholesterol, and BFP. NAFLD and NAPS cases had significant differences in age ( $44 \pm 13$ vs $56 \pm 15, \mathrm{p}<0.001)$, BMI (28.1 \pm 4.2 vs $26.5 \pm 2.7, \mathrm{p}=0.04)$, and HDL-cholesterol (46.0 \pm 9.1 vs $54 \pm 13, \mathrm{p}=0.01$ ), respectively (Table 2 ). 
Table I Demographic and Anthropometric Features of Included Patients

\begin{tabular}{|l|l|l|l|l|l|}
\hline Status of Steatosis & Normal & NAFLD & NAPS & NAPS+NAFLD & P** \\
\hline Cases $(\mathrm{n}=345)$ & $74(21.4 \%)$ & $44(12,8 \%)$ & $52(15.1 \%)$ & $175(50,7 \%)$ & - \\
Sex (F/M) & $54 / 20$ & $29 / 15$ & $37 / 15$ & $100 / 75$ & 0.661 \\
Age (years) & $41 \pm 14$ & $44 \pm 13$ & $56.4 \pm 15$ & $55 \pm 13$ & $0.000^{*}$ \\
BMI (kg/m $\left.{ }^{2}\right)$ & $23 \pm 3$ & $28 \pm 4$ & $26 \pm 3$ & $31 \pm 5$ & $0.040 *$ \\
BFP $(\%)$ & $28 \pm 8$ & $34 \pm 9$ & $34 \pm 7$ & $39 \pm 9$ & 0.930 \\
Diabetes mellitus & $5(6.8 \%)$ & $7(15,9 \%)$ & $9(17.3 \%)$ & $84(36 \%)$ & 1.000 \\
Hepatomegaly & $24,3 \%$ & $47.7 \%$ & $30.8 \%$ & $70.3 \%$ & 0.098 \\
Cholecystolithiasis & $6,8 \%$ & $13,6 \%$ & $28.8 \%$ & $26.3 \%$ & 0.087 \\
\hline
\end{tabular}

Notes: *Significant $(\mathrm{p}<0.05)$; **Comparison between NAFLD and NAPS.

Abbreviations: NAFLD, nonalcoholic fatty liver disease; NAPS, nonalcoholic pancreatic steatosis; F, female; M, male; BMI, body mass index; BFP, body fat percentage.

Table 2 Laboratory Values of the Study Group

\begin{tabular}{|l|l|l|l|l|l|}
\hline Status of Steatosis & Normal & NAFLD & NAPS & NAPS+NAFLD & p** \\
\hline Insulin level & $6.6 \pm 2.3$ & $8.3 \pm 4.0$ & $9.6 \pm 8.0$ & $13.5 \pm 8.9$ & $0.000^{*}$ \\
Insulin resistance & $1.5 \pm 0.6$ & $1.9 \pm 0.8$ & $3.2 \pm 5.7$ & $3.9 \pm 3.5$ & 0.017 \\
ALT (IU/mL) & $22 \pm 19$ & $29 \pm 18$ & $23 \pm 21$ & $31 \pm 23$ & 0.130 \\
Triglyceride $(\mathrm{mg} / \mathrm{dL})$ & $93 \pm 47$ & $137 \pm 65$ & $119 \pm 76$ & $164 \pm 83$ & 0.218 \\
HDL-cholesterol & $53 \pm 13$ & $46 \pm 9$ & $54 \pm 13$ & $48 \pm 15$ & $0.00 I^{*}$ \\
Total cholesterol & $189 \pm 47$ & $205 \pm 46$ & $202 \pm 42$ & $207 \pm 45$ & 0.816 \\
\hline
\end{tabular}

Notes: *Significant $(p<0.05)$; $* *$ Comparison between NAFLD and NAPS.

Abbreviations: NAFLD, nonalcoholic fatty liver disease; NAPS, nonalcoholic pancreatic steatosis; ALT, alanine aminotransferase.

\section{Discussion}

NAFLD and fatty infiltration of the pancreas are associated with abdominal obesity, insulin resistance, dyslipidemia, diabetes mellitus, and hypertension and are significant causes of global ill health and economic burden., ${ }^{3,5}$ The adipose tissue, as ectopic fat, may accumulate in the pancreas, muscles, renal sinus of the kidneys, cardiovascular system, internal organs, etc. ${ }^{12-14}$ Nonalcoholic pancreatic steatosis is also identified as fatty pancreas, nonalcoholic fatty pancreas disease, lipomatous pseudohypertrophy, and pancreatic lipomatosis. ${ }^{15,16}$ In this paper, we chose NAPS for clarity. Abdominal US was used in diagnosing and grading of NAPS and NAFLD. A previous study reported that US could better detect NAPS than NAFLD. ${ }^{17}$ Although noninvasive methods for detection and quantification with imaging techniques are being evaluated, there is still a lack of standardization. ${ }^{18,19}$

The role of NAPS is still debatable in acute and chronic pancreatitis, pancreatic cancer, and pancreatic exocrine insufficiency. ${ }^{20}$ Older age, high BMI, and insulin resistance are reportedly associated with NAPS, ${ }^{9,12,21,22}$ although some studies have found no relation between NAPS and beta-cell function. ${ }^{23}$ Fat infiltration of the pancreas may lead to more bleeding and post-operative fistula formation in pancreatic surgery. ${ }^{15,24}$ NAPS was reported to be associated with prediabetes, especially in male subjects. ${ }^{25}$ In our study, compared to NAFLD, older age, higher HDL-cholesterol, and higher IR were more associated with NAPS, whereas triglycerides (Figure 1), diabetes mellitus, and BMI were prominent in NAFLD cases, consistent with some reports. ${ }^{5,25-27}$

The proposed mechanisms of fatty infiltration in the pancreas were the substitution of acinar cells by adipose tissue and the adipocyte effect on exocrine function. In NAFLD, triglycerides and fatty acids are stored within the hepatocytes, whereas adipocytes and pancreatic fat accumulate partly in acinar cells and mainly in the intralobular and interlobular area, especially in septae which are horizontally parallel to the main duct and primarily in the pancreatic head. ${ }^{2,12,28}$ Both ongoing fibrosis and replacement of functional cells of the pancreas with fat and adipocytes may result in insufficiencies in endocrine and exocrine functions. ${ }^{29,30}$ Other data have shown that NAPS affects exocrine functions more than endocrine cells and is associated with age, BMI, and decreased serum lipase. ${ }^{31}$ Our results for amylase and lipase were not different between NAPS patients with normal and steatotic livers $(p=0.793)$. 


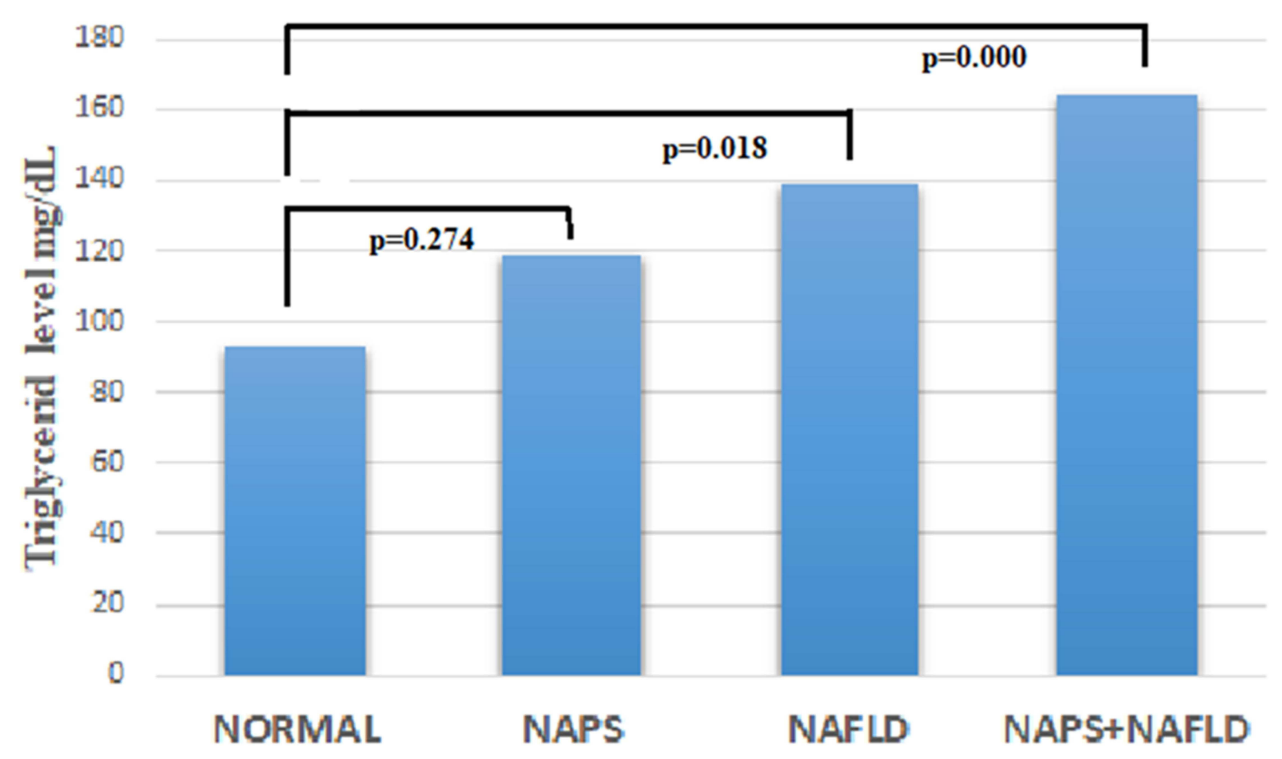

Figure I Serum Triglyceride levels of subgroups.

Abbreviations: NAPS, Nonalcoholic pancreatic steatosis; NAFLD, Nonalcoholic fatty liver disease.

The strong correlation of cholecystolithiasis with NAFLD and NAPS in cholecystectomy has been reported in a previous study. ${ }^{32}$ In our study, gall bladder diseases were highly associated with NAPS rather than NAFLD. The pancreas and gallbladder originate from the same endodermal pouch and are bound via the common bile duct and Wirsung channel. As in biliary pancreatitis, the inflammatory storm or low-level ongoing inflammation in obesity may simultaneously affect both organs. Moreover, age and obesity as the shared risk factors for both may explain the simultaneous increase in fatty pancreas and cholecystolithiasis.

The mechanism of excess fat accumulation may be pathophysiologically different between alcoholic and metabolic fatty pancreas. In a study, alcohol caused intra-pancreatic esterification of cholesterol and thereby accumulation of cholesteryl ester but not other lipids in three-week alcohol- or isocaloric carbohydrate-fed rats. ${ }^{33}$ NAPS may progress to nonalcoholic steatopancreatitis (NASP), and analyzing these two consequential steps separately, as in the relationship between NAFLD and nonalcoholic steatohepatitis (NASH), may give more uniform results. The effect of adipocytesderived adipokines, macrophages, metabolites, inflammatory cytokines, and pro-oncogenic integrals of fatty pancreas infiltration have not been fully explained. ${ }^{13,34-36}$ In our study, $16.1 \%$ of NAFLD had NASH, roughly based on elevated ALT, but no additional risk on presence of NAPS ( $\mathrm{p}=0.921$ ). In cases with steatosis, $19.2 \%$ had NAPS without NAFLD (Figure 2). As a multifactorial clinical benign entity, the coexistence of NAPS and NAFLD may also be the result of them sharing similar risk factors. The main message of this study is the necessity of subgrouping the fatty pancreas as isolated NAPS and NAFLD+NAPS in future studies, since distinct differences in demographic, anthropometric, and biochemical features exist. Recent data suggest subgroups in NAFLD, too. NAFLD and metabolic associated fatty liver (MAFLD) cover different descriptions; for the latter, the presence of at least two metabolic abnormal components is mandatory. ${ }^{39}$

This study has some limitations. First, US is highly operator-dependent, although the same radiologist evaluated the cases. Ultrasound has limitations in differentiating pancreatic steatosis and fibrosis, as both may reflect echogenic pancreas. ${ }^{15}$ Computed tomography, endoscopic US, magnetic resonance imaging, and MR-based proton density fat fraction are some of the preferred imaging modalities for more standardized data, according to some published reports on prospective studies. ${ }^{22,34,37,38}$ The included cases are patients admitted to the hospital; thus, they may not represent the overall population. Furthermore, ethnic, geographic, and nutritional comparisons were not performed. The study's retrospective design is another limitation, resulting in missing details about anthropometric, past history and viral serology data.

\section{Conclusion}

In this study, nonalcoholic pancreatic steatosis did not fully correlate with non-alcoholic fatty liver based on ultrasound, clinical, demographic, and anthropometric features. Despite severe pancreatic steatosis, more than a quarter of cases had completely normal liver echogenicity. In NAPS, compared 


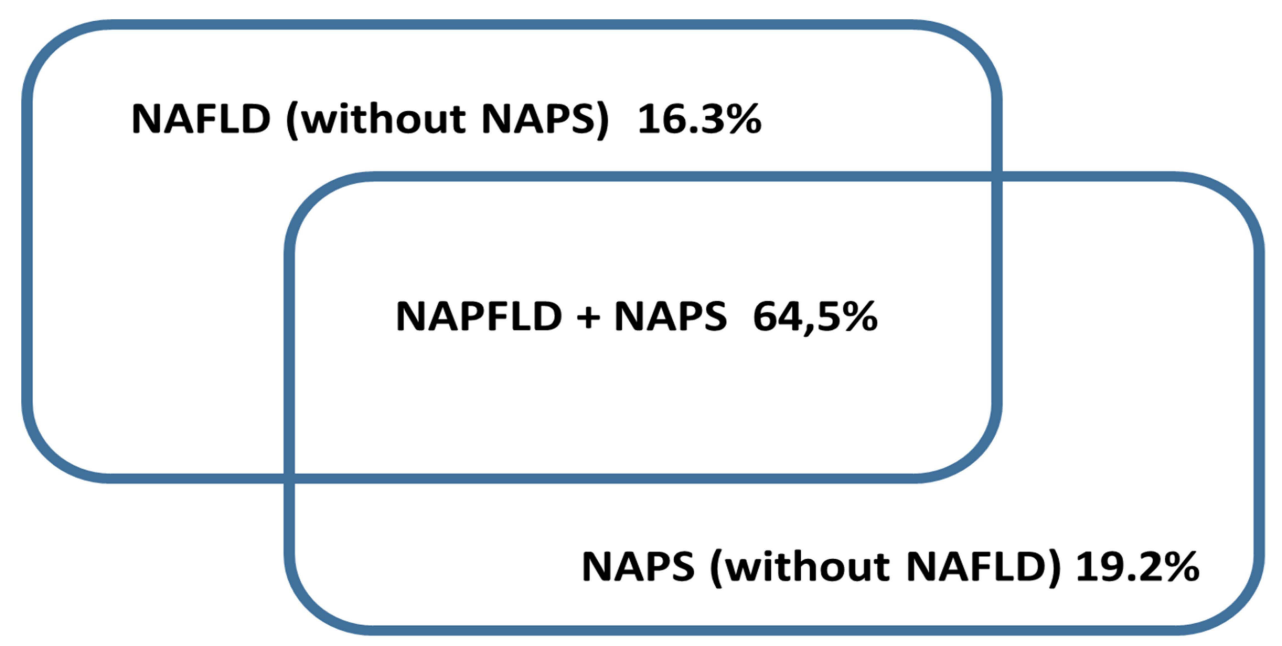

Figure 2 Venn diagram of NAPS and NAFLD.

Abbreviations: NAPS, Nonalcoholic pancreatic steatosis; NAFLD, Nonalcoholic fatty liver disease.

to NAFLD, insulin resistance and HDL-cholesterol were The presence of a higher frequency of cholecystolithiasis in insignificantly higher, whereas BMI, total cholesterol, BFP, NAPS needs further investigation in a larger study. This LDL-cholesterol, and triglycerides were lower (Figure 3). inconsistency in $>50 \%$ grading of liver and pancreatic

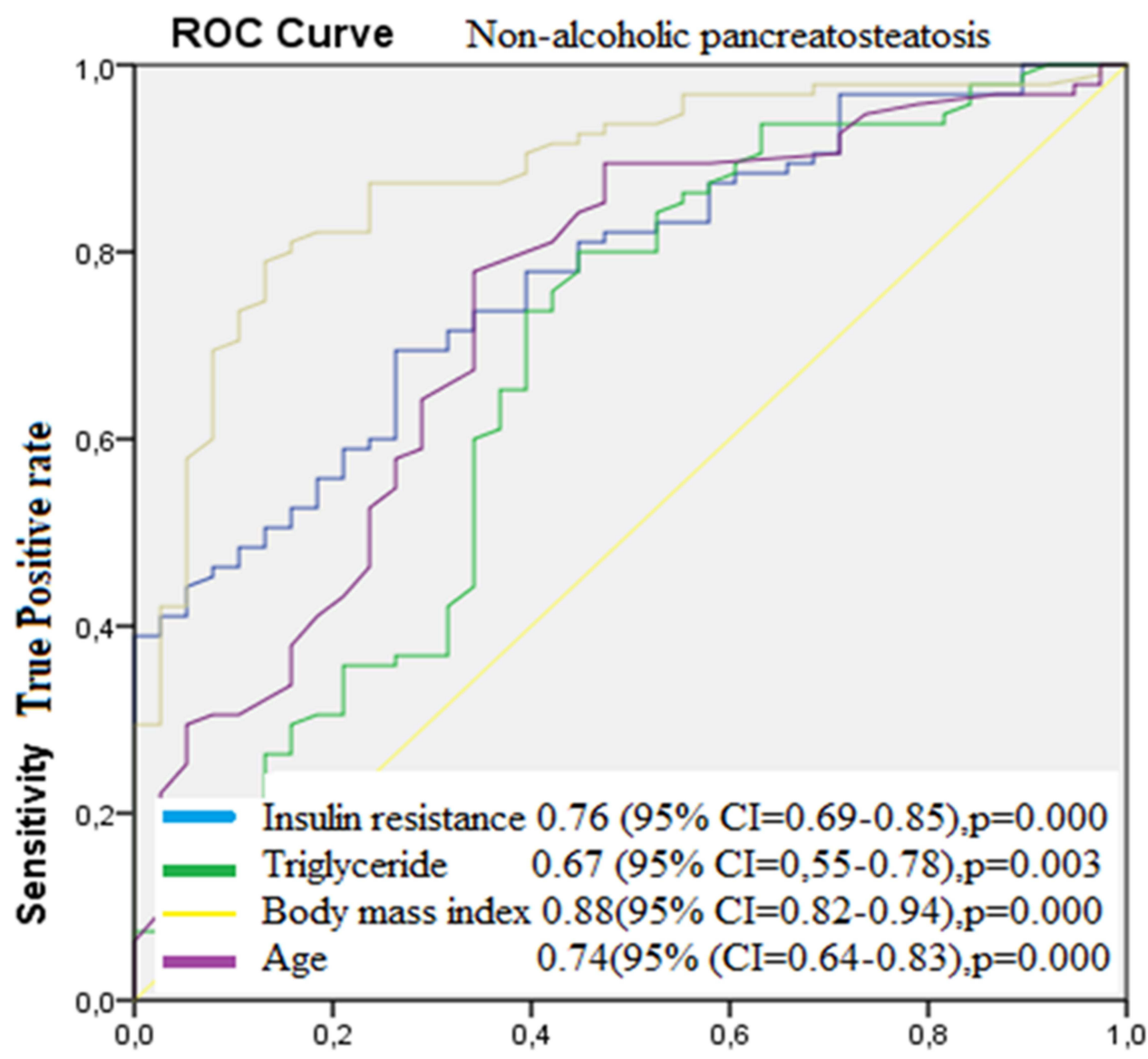

\section{1 - Specificity False negative rate}

Figure 3 Receiver operating characteristic (ROC) curve for insulin resistance, triglyceride, body mass index, and age in nonalcoholic pancreatic steatosis area under the ROC curve. 
steatosis may reflect different pathophysiological components of these clinical entities.

\section{Disclosure}

The authors reported no conflicts of interest in this work.

\section{References}

1. Alempijevic T, Dragasevic S, Zec S, Popovic D, Milosavljevic T. Non-alcoholic fatty pancreas disease. Postgrad Med J. 2017;93 (1098):226-230. doi:10.1136/postgradmedj-2016-134546

2. Dite P, Blaho M, Bojkova M, Jabandziev P, Kunovsky L. Nonalcoholic fatty pancreas disease: clinical consequences. Dig Dis. 2020;38(2):143-149. doi:10.1159/000505366

3. Majumder S, Philip NA, Takahashi N, Levy MJ, Singh VP, Chari ST. Fatty pancreas: should we be concerned? Pancreas. 2017;46 (10):1251-1258. doi:10.1097/MPA.0000000000000941

4. Mirrakhimov AE. Nonalcoholic fatty pancreatic disease and cardio-metabolic risk: is there is a place for obstructive sleep apnea? Cardiovasc Diabetol. 2014;13:29. doi:10.1186/1475-284013-29

5. Tirkes T, Jeon CY, Li L, et al. Association of pancreatic steatosis with chronic pancreatitis, obesity, and type 2 diabetes mellitus. Pancreas. 2019;48(3):420-426. doi:10.1097/MPA.0000000000001252

6. van Geenen EJ, Smits MM, Schreuder TC, van der Peet DL, Bloemena E, Mulder CJ. Nonalcoholic fatty liver disease is related to nonalcoholic fatty pancreas disease. Pancreas. 2010;39 (8):1185-1190. doi:10.1097/MPA.0b013e3181f6fce2

7. Gerst F, Wagner R, Kaiser G, et al. Metabolic crosstalk between fatty pancreas and fatty liver: effects on local inflammation and insulin secretion. Diabetologia. 2017;60(11):2240-2251. doi:10.1007/ s00125-017-4385-1

8. Lesmana CR, Pakasi LS, Inggriani S, Aidawati ML, Lesmana LA. Prevalence of Non-Alcoholic Fatty Pancreas Disease (NAFPD) and its risk factors among adult medical check-up patients in a private hospital: a large cross-sectional study. BMC Gastroenterol. 2015;15:174. doi:10.1186/s12876-015-0404-1

9. So CB, Cooperberg PL, Gibney RG, Bogoch A. Sonographic findings in pancreatic lipomatosis. AJR Am J Roentgenol. 1987;149(1):67-68. doi:10.2214/ajr.149.1.67

10. Zsori G, Ills D, Ivony E, et al. In new-onset diabetes mellitus, metformin reduces fat accumulation in the liver, but not in the pancreas or pericardium. Metab Syndr Relat Disord. 2019;17 (5):289-295. doi:10.1089/met.2018.0086

11. Ferraioli G, Soares Monteiro LB. Ultrasound-based techniques for the diagnosis of liver steatosis. World J Gastroenterol. 2019;25 (40):6053-6062. doi:10.3748/wjg.v25.i40.6053

12. Takahashi M, Hori M, Ishigamori R, Mutoh M, Imai T, Nakagama H. Fatty pancreas: a possible risk factor for pancreatic cancer in animals and humans. Cancer Sci. 2018;109(10):3013-3023. doi:10.1111/ cas. 13766

13. Kozawa J, Shimomura I. Ectopic fat accumulation in pancreas and heart. J Clin Med. 2021;10(6):1326. doi:10.3390/jcm 10061326

14. Kim MK, Chun HJ, Park JH, et al. The association between ectopic fat in the pancreas and subclinical atherosclerosis in type 2 diabetes. Diabetes Res Clin Pract. 2014;106(3):590-596. doi:10.1016/j. diabres.2014.09.005

15. Catanzaro R, Cuffari B, Italia A, Marotta F. Exploring the metabolic syndrome: nonalcoholic fatty pancreas disease. World $J$ Gastroenterol. 2016;22(34):7660-7675. doi:10.3748/wjg.v22. i34.7660

16. Tariq H, Nayudu S, Akella S, Glandt M, Chilimuri S. Non-alcoholic fatty pancreatic disease: a review of literature. Gastroenterology Res. 2016;9(6):87-91. doi:10.14740/gr731w
17. Li S, Su L, Lv G, Zhao W, Chen J. Transabdominal ultrasound of the pancreas is superior to that of the liver for detection of ectopic fat deposits resulting from metabolic syndrome. Medicine (Baltimore). 2017;96(37):e8060. doi:10.1097/MD.0000000000008060

18. Lingvay I, Esser V, Legendre JL, et al. Noninvasive quantification of pancreatic fat in humans. J Clin Endocrinol Metab. 2009;94 (10):4070-4076. doi:10.1210/jc.2009-0584

19. Milovanovic T, Dragasevic S, Stojkovic Lalosevic M, et al. Ultrasonographic evaluation of fatty pancreas in serbian patients with non alcoholic fatty liver disease-a cross sectional study. Medicina (Kaunas). 2019;55(10):697. doi:10.3390/medicina55100697

20. Kromrey ML, Friedrich N, Hoffmann RT, et al. Pancreatic steatosis is associated with impaired exocrine pancreatic function. Invest Radiol. 2019;54(7):403-408. doi:10.1097/RLI.0000000000000554

21. Fujii M, Ohno Y, Yamada M, Kamada Y, Miyoshi E. Impact of fatty pancreas and lifestyle on the development of subclinical chronic pancreatitis in healthy people undergoing a medical checkup. Environ Health Prev Med. 2019;24(1):10. doi:10.1186/s12199-019-0763-2

22. Al-Mrabeh A, Hollingsworth KG, Steven S, Tiniakos D, Taylor R. Quantification of intrapancreatic fat in type 2 diabetes by MRI. PLoS One. 2017;12(4):e0174660. doi:10.1371/journal.pone.0174660

23. Begovatz P, Koliaki C, Weber K, et al. Pancreatic adipose tissue infiltration, parenchymal steatosis and beta cell function in humans. Diabetologia. 2015;58(7):1646-1655. doi:10.1007/s00125-015-3544-5

24. Smits MM, van Geenen EJ. The clinical significance of pancreatic steatosis. Nat Rev Gastroenterol Hepatol. 2011;8(3):169-177. doi:10.1038/nrgastro.2011.4

25. Ou HY, Wang CY, Yang YC, Chen MF, Chang CJ. The association between nonalcoholic fatty pancreas disease and diabetes. PLoS One. 2013;8(5):e62561. doi:10.1371/journal.pone.0062561

26. Patel NS, Peterson MR, Lin GY, et al. Insulin resistance increases MRI-estimated pancreatic fat in nonalcoholic fatty liver disease and normal controls. Gastroenterol Res Pract. 2013;2013:498296. doi:10.1155/2013/498296

27. Rossi AP, Fantin F, Zamboni GA, et al. Predictors of ectopic fat accumulation in liver and pancreas in obese men and women. Obesity (Silver Spring). 2011;19(9):1747-1754. doi:10.1038/oby.2011.114

28. Worthen NJ, Beabeau D. Normal pancreatic echogenicity: relation to age and body fat. AJR Am J Roentgenol. 1982;139(6):1095-1098. doi:10.2214/ajr.139.6.1095

29. Apte MV, Wilson JS, Korsten MA. Alcohol-related pancreatic damage: mechanisms and treatment. Alcohol Health Res World. 1997;21(1):13-20.

30. Coulier B. Pancreatic lipomatosis: an extensive pictorial review. J Belg Soc Radiol. 2016;100(1):39. doi:10.5334/jbr-btr.1014

31. Kuhn JP, Berthold F, Mayerle J, et al. Pancreatic steatosis demonstrated at MR imaging in the general population: clinical relevance. Radiology. 2015;276(1):129-136. doi:10.1148/radiol.15140446

32. Koc U, Taydas O. Investigation of the relationship between fatty pancreas and cholecystectomy using noncontrast computed tomography. J Med Imaging Radiat Sci. 2019;50(2):220-226. doi:10.1016/j.jmir.2018.10.007

33. Wilson JS, Colley PW, Sosula L, Pirola RC, Chapman BA, Somer JB. Alcohol causes a fatty pancreas. A rat model of ethanol-induced pancreatic steatosis. Alcohol Clin Exp Res. 1982;6 (1):117-121. doi:10.1111/j.1530-0277.1982.tb05389.x

34. Sakai NS, Taylor SA, Chouhan MD. Obesity, metabolic disease and the pancreas-quantitative imaging of pancreatic fat. $\mathrm{Br} J$ Radiol. 2018;91(1089):20180267. doi:10.1259/bjr.20180267

35. Gerst $\mathrm{F}$, Wagner R, Oquendo MB, et al. What role do fat cells play in pancreatic tissue? Mol Metab. 2019;25:1-10. doi:10.1016/j. molmet.2019.05.001

36. Khoury T, Asombang AW, Berzin TM, Cohen J, Pleskow DK, Mizrahi M. The clinical implications of fatty pancreas: a concise review. Dig Dis Sci. 2017;62(10):2658-2667. doi:10.1007/s10620017-4700-1 
37. Choi CW, Kim GH, Kang DH, et al. Associated factors for a hyperechogenic pancreas on endoscopic ultrasound. World J Gastroenterol. 2010;16(34):4329-4334. doi:10.3748/wjg.v16.i34.4329

38. Heber SD, Hetterich H, Lorbeer R, et al. Pancreatic fat content by magnetic resonance imaging in subjects with prediabetes, diabetes, and controls from a general population without cardiovascular disease. PLoS One. 2017;12(5):e0177154. doi:10.1371/journal.pone.0177154
39. Godoy-Matos AF, Silva WS, Valerio CM. NAFLD as a continuum: from obesity to metabolic syndrome and diabetes. Diabetol Metab Syndr. 2020;12:60. doi:10.1186/s13098-020-00570-y

\section{Publish your work in this journal}

Clinical and Experimental Gastroenterology is an international, peerreviewed, open access, online journal publishing original research, reports, editorials, reviews and commentaries on all aspects of gastroenterology in the clinic and laboratory. This journal is indexed on American Chemical Society's Chemical Abstracts Service (CAS)
The manuscript management system is completely online and includes a very quick and fair peer-review system, which is all easy to use. Visit http://www.dovepress.com/testimonials.php to read real quotes from published authors. 